\title{
Testing gravitation with satellite laser ranging and the LARASE experiment
}

\author{
David M. Lucchesi ${ }^{a, b, c, *}$, Luciano Anselmo ${ }^{c}$, Massimo Bassan $^{d, b}$, Carmen Pardini $^{c}$, \\ Roberto Peron $^{a, b}$, Giuseppe Pucacco ${ }^{d, b}$ and Massimo Visco ${ }^{a, b}$ \\ ${ }^{a}$ Istituto Nazionale di Astrofisica (INAF), Istituto di Astrofisica e Planetologia Spaziali (IAPS) \\ Via Fosso del Cavaliere, 100, 00133 Roma, Italy \\ *E-mail: david.lucchesi@iaps.inaf.it \\ ${ }^{b}$ Istituto Nazionale di Fisica Nucleare (INFN), sezione di Roma Tor Vergata \\ Via della Ricerca Scientifica, 1, 00133 Roma, Italy \\ ${ }^{c}$ Istituto di Scienza e Tecnologie della Informazione (ISTI), Consiglio Nazionale delle Ricerche \\ $(C N R)$ \\ Via Moruzzi, 1, 56124 Pisa, Italy \\ ${ }^{d}$ Dipartimento di Fisica, Universitá di Roma Tor Vergata \\ Via della Ricerca Scientifica, 1, 00133 Roma, Italy
}

The International Laser Ranging Service (ILRS) provides range measurements of passive satellites around the Earth through the powerful Satellite Laser Ranging (SLR) technique. These very precise measurements of the distance between an on-ground laser station and a satellite equipped with cube corner retro-reflectors (CCRs) make possible precise tests and measurements in fundamental physics and, in particular, in gravitational physics. The LAGEOS (NASA 1976) and LAGEOS II (NASA/ASI 1992) satellites are outstanding examples of very good test particles because of their very low area-to-mass ratio as well as the high quality of their tracking data and, consequently, of the precise orbit determination (POD) we can obtain after a refined modeling of their orbit. The aim of our research program LARASE (LAser RAnged Satellites Experiment) is to go a step further in testing gravitation in the field of Earth by means of the joint analysis of the orbits of the two LAGEOS satellites together with that of the most recently launched LARES (ASI, 2012) satellite. Therefore, our work falls in the so-called weak field and slow motion (WFSM) limit of Einstein's general relativity (GR) where, in terms of Newtonian physics, relativistic effects appear as two new fields to be added to the classical gravitational field: the gravitoelectric and the gravitomagnetic fields. A fundamental ingredient to reach such a goal is to provide high-quality updated models for the perturbing non-gravitational perturbations (NGP) acting on the surface of these satellites. In fact, regardless of their minimization thanks to a smaller value for the area-to-mass ratio, the subtle and complex to model perturbing effects of the NGP play a crucial role in the POD of the considered satellites, especially in the case of the thermal thrust effects. A large amount of SLR data of LAGEOS and LAGEOS II has been worked out using a set of dedicated models for the satellite dynamics and the related post-fit residuals have been analyzed. A parallel work was performed with LARES, although at a preliminary stage. Our recent work on the orbit modeling and on the data analysis of the orbit of such satellites is presented and discussed.

Keywords: General Relativity measurements; laser-ranged satellites; gravitational and non-gravitational perturbations; MG14 Proceedings. 


\section{Introduction}

Nowadays, after 100 years from its formulation, Einstein's theory of general relativity (GR) still represents the best theory at our disposal for the description of gravity and of its complex interaction ${ }^{1}$. Such description works very well both at high and low energy scales. Indeed, GR has passed a wide number of experimental and observational verifications during the last 50 years ${ }^{2}$, with both direct and indirect manifestations of its predictions. Therefore, today, GR represents the Standard Model for the description of the gravitational interaction, and it is the pillar of modern cosmology to understand the universe that we observe.

GR represents a metric theory of gravity and it is fully described in terms of a metric tensor $g_{\mu \nu}$. However, after its formulation, other gravitational theories have been proposed. Some of these are metric theories, hence they share with GR the same spacetime structure and the same equations of motion for test particles, but differ in the field equations form. Very importantly, Einstein Equivalence Principle $(\mathrm{EEP})^{3,4}$ is at the basis of GR and of any metric theory of gravity.

Conversely, other theories provide more fundamental differences, such as violations of EEP, and fall in the family of the non-metric theories. These non-metric theories are characterized by additional fields, beside the metric tensor $g_{\mu \nu}$ of GR, that act as "new" gravitational fields. These additional fields may be scalar, vectorial or tensorial in their nature. Their role is to "explain" how the matter and the non-gravitational fields generate the gravitational fields themselves and produce the metric.

Furthermore, Einstein's theory of GR is a classical theory of physics and all the attempts at merging gravitation with the other interactions of nature have failed up to now. In fact, the overall validity of Einstein's GR has been questioned by quantum theories of physics. Indeed, the (possible) existence of additional fields in mediating the gravitational interaction is also predicted by modern theories of physics — besides the cited alternative theories of gravity — which aim to unify gravity with the quantum realm. Therefore, it is clear, as a consequence of the above (general) considerations, that it is very important to precisely test the consequences of GR, as well as those of competing theories, at all the accessible scales of distances and energies.

The rest of the paper is organized as follows. In Section 2, the main goals of LARASE will be highlighted together with a brief description of the main relativistic precession to be measured. In Section 3, we briefly describe the setup and the models of the software used for the data reduction of the orbit of the satellites and we give our preliminary results for their precise orbit determination in terms of the rootmean-square (RMS) of the range residuals. In Section 4, the improvements that we have reached in the models we analyzed so far for the NGP are described. Finally, Section 5 is devoted to our conclusions and recommendations. 


\section{LARASE Goals and GR Relativistic Precessions}

The LAser RAnged Satellites Experiment (LARASE) ${ }^{5}$ aims to provide an original contribution in testing and verifying gravitation in the weak-field and slow-motion limit (WFSM) of Einstein's GR. The final goal of LARASE is to provide a refined and reliable error budget for each precise measurement of a relativistic effect. Such objectives will be reached thanks to a precise orbit determination (POD) of a ded-

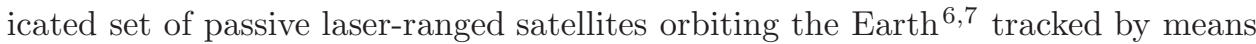
of the powerful Satellite Laser Ranging (SLR) technique of the International Laser Ranging Service ${ }^{8}$.

A major goal of LARASE is to improve the dynamical models of the current best laser-ranged satellites, with a special attention to the subtle and complex to model non-gravitational perturbations (NGP). Indeed, in order to obtain a refined POD, beside high quality tracking observations of the satellite, it is necessary a reliable dynamical model for the various perturbations to be included in the software for the orbit determination of the satellite.

The proof-masses of the LARASE experiment are constituted by the best laserranged satellites orbiting the Earth. Among them, the two LAGEOS and the recently launched LARES are the most important to consider because of the high accuracy of their orbit determination. The older LAGEOS (LAser GEOdynamic Satellite) was launched by NASA on May 4, 1976, LAGEOS II was jointly launched by NASA and ASI on October 22, 1992, finally LARES (LAser RElativity Satellite) was launched by ASI on February 13, 2012. These satellites are spherical in shape, fully passive, and with a generally low area-to-mass ratio in order to minimize the non-gravitational accelerations. In particular, LARES is the densest object ever launched by the man in space, with an area-to-mass ratio 2.6 times smaller than that of the two LAGEOS satellites ${ }^{9,10}$.

Concerning the measurements to be performed, LARASE will mainly focus on the relativistic precessions related with the Earth's gravitoelectric and gravitomagnetic fields. The former field, analogous to the electric field due to electric charges of Coulomb's law, is produced by the Earth's mass, while the latter, analogous to the magnetic field due to electric currents, is produced by the Earth's current-of-mass, i.e., by the Earth's angular momentum.

These relativistic precessions are, respectively, Einstein (or Schwarzschild) ${ }^{1}$, and Lense-Thirring (LT) ${ }^{11,12}$ precessions. These precessions are responsible of long-term and secular effects on two of the three Euler angles that define the orbit orientation in space, namely the argument of pericenter, $\omega$, which is subject to both precessions, and the right ascension of the ascending node, $\Omega$, which is subject to the LenseThirring one.

For the secular effects we have the following expressions:

$$
\dot{\omega}^{\mathrm{Schw}}=\frac{3\left(G M_{\oplus}\right)^{3 / 2}}{c^{2} a^{5 / 2}\left(1-e^{2}\right)},
$$


for the gravitoelectric precession of the argument of pericenter, and

$$
\dot{\omega}^{\mathrm{LT}}=\frac{-6 G J_{\oplus}}{c^{2} a^{3}\left(1-e^{2}\right)^{3 / 2}} \cos i,
$$

for its gravitomagnetic part, and

$$
\dot{\Omega}^{\mathrm{LT}}=\frac{2 G J_{\oplus}}{c^{2} a^{3}\left(1-e^{2}\right)^{3 / 2}},
$$

for the gravitomagnetic precession of the satellite node.

In the above equations, $G$ and $c$ are, respectively, the gravitational constant and the speed of light, $M_{\oplus}$ and $J_{\oplus}$ represent the mass and angular momentum of the Earth, finally, $a, e$ and $i$, are, respectively, the orbit semi-major axis, eccentricity and inclination. In Table 1, the computed values of the relativistic precession for the LAGEOS and LARES satellites are shown.

Table 1. Rate (mas/yr) for the gravitoelectric and gravitomagnetic secular precession for the two LAGEOS satellites and LARES ( 1 mas/yr $=1$ milli-arc-second per year).

\begin{tabular}{cccc}
\hline Rate $[\mathrm{mas} / \mathrm{yr}]$ & LAGEOS & LAGEOS II & LARES \\
\hline$\dot{\omega}^{\mathrm{Shw}}$ & 3278.78 & 3352.58 & 10110.13 \\
$\dot{\omega}^{\mathrm{LT}}$ & 31.23 & -57.33 & -124.53 \\
$\dot{\Omega}^{\mathrm{LT}}$ & 30.67 & 31.51 & 118.47 \\
\hline
\end{tabular}

Since a way to test the predictions of Einstein's GR with respect to those of other metric theories is through the measurements of the so-called parameterized post-Newtonian $(\mathrm{PPN})$ parameters ${ }^{13-16}$, a few of them (namely $\gamma, \beta, \alpha_{1}$ and $\alpha_{2}$ ) will be the subject of the investigations and measurements of LARASE.

\section{Precise Orbit Determination}

In order to perform reliable measurements in gravitational physics by means of the tracking of Earth's satellites ${ }^{17-25}$, as well as for space geodesy and geophysics applications $^{26-32}$, a POD for the orbit of the considered satellites represents an essential prerequisite. In this context, the preliminary analyses we performed include a preparatory data reduction for the satellites orbit with a tailored setup for the models implemented in the software.

For the data reduction, i.e., the differential correction procedure to obtain the POD of the satellites, we used the GEODYN II software of NASA/GSFC ${ }^{7}$. In Table 2, the models currently implemented in GEODYN II that we used in our data reductions are shown. These models include the GR corrections in the PPN formalism. Of course, such corrections need to be removed from the setup in order to recover the secular precessions provoked by GR in the residuals time series.

The state vector of the satellites, with selected station biases, the radiation coefficient and the corrections to polar motion and length of day have been estimated. 
Table 2. Modelling setup as included in the analysis. Three groups are indicated: gravitational effects, non-gravitational ones and reference frames.

\begin{tabular}{lll}
\hline Model for & Model type & Reference \\
\hline Geopotential (static) & EGM96, EIGEN-GRACE02S & 30,33 \\
Geopotential (time-varying, tides) & Ray GOT99.2 & 34 \\
Geopotential (time-varying, non tidal) & IERS Conventions (2010) & 35 \\
Third-body & JPL DE-403 & 36 \\
Relativistic corrections & Parameterized post-Newtonian & 37 \\
\hline Direct solar radiation pressure & Cannonball & 7 \\
Earth albedo & Knocke-Rubincam & 38 \\
Earth-Yarkovsky & Rubincam (1987-1990) & $39-41$ \\
Neutral drag & NRLMSISE-2000 & 42 \\
Spin & LARASE (2014-2016) & To be published \\
\hline Stations position & ITRF2008 & 43 \\
Ocean loading & Schernek and GOT99.2 tides & 7,34 \\
Earth Rotation Parameters & IERS EOP C04 & 44 \\
\hline
\end{tabular}

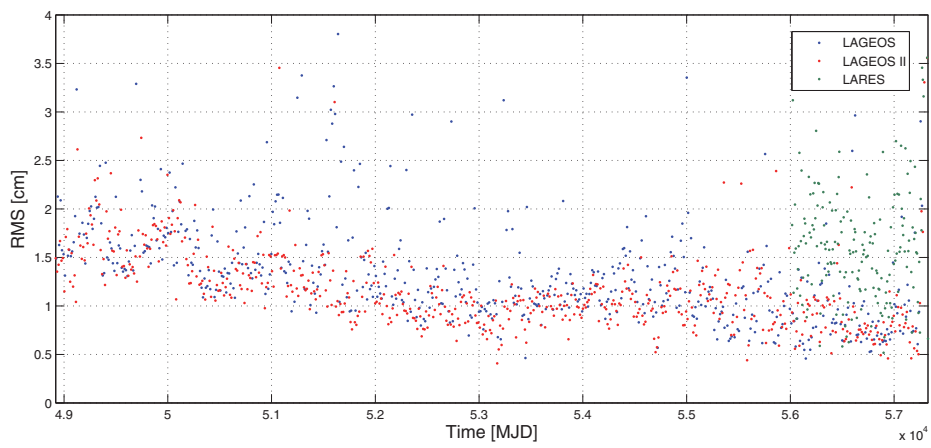

Fig. 1. Root Means Square (RMS) of the range residuals of LAGEOS (blue), LAGEOS II (red) and LARES (green), the units are in centimeters. The time is given in Modified Julian Date (MJD). In the case of the two LAGEOS, the starting epoch (MJD 48919) for the POD corresponds to October 24, 1992, while, in the case of LARES, the starting epoch (MJD 56017) corresponds to March 31, 2012. The arc length is 14 days for LAGEOS and LAGEOS II, and 7 days for LARES. Notice the higher uncertainty associated with the LARES analysis, showing its currently non-optimal modelling.

In these analyses, empirical accelerations have been also used. The results of these preliminary analyses for the three satellites are shown in Figure 1.

As we can see, LAGEOS and LAGEOS II orbits are recovered with a mean error roughly between 1.5 and $1 \mathrm{~cm}$, while the orbit of LARES has a slightly higher error due to a currently yet non-optimal modelling for its dynamics. Finally, it is important to highlight that the decreasing trend that we obtained for the RMS of the range residuals in the case of the two LAGEOS, which approaches the $5 \mathrm{~mm}$ (mean) value at the end of the time span, is in good agreement with the RMS obtained from the data reduction of the orbit of the two LAGEOS satellites performed by the main Analysis Centers of the ILRS network. 


\section{Improvements in the Modeling of the Satellites' Orbit}

A reliable modeling of the gravitational and NGP is mandatory in order to reach a very good POD for the considered satellites and then extract from their orbital residuals ${ }^{45}$ the imprint of the GR effects.

With regard to the gravitational perturbations, a key role is played by the uncertainties of the even zonal harmonics coefficients of the Earths's gravitational field expansions in spherical harmonics. In fact, an error in the values of such coefficients is responsible of a residual (unmodeled) secular effect — both on the right ascension of the ascending node $\Omega$ and on the argument of pericenter $\omega$ - that corrupts the measurement of the corresponding relativistic precession. Moreover, the low degree $\ell$ coefficients, i.e. those to which the two LAGEOS are most sensitive to, are also time-dependent, and this represents a further complication to be taken into account properly. The tides are also important in the final error budget, in particular for a POD performed over a short time span compared with the period of the main tidal perturbations. Such effects, and their uncertainties, are especially important for LARES, because of its much lower height (about $1450 \mathrm{~km}$ ) with respect to that of the two LAGEOS (about $5900 \mathrm{~km}$ ).

However, as stated in Section 2, the major difficulties in the modeling are connected with the NGP, because of their intrinsic complexity and their corresponding subtle effects on the orbit of a satellite. In particular, the thermal thrusts perturbations, as in the case of the Yarkovsky-Schach effect $\left(\mathrm{see}^{38,40,46-54}\right)$, are responsible, if not properly modeled, of long-term effects on the orbital elements, as in the case of the argument of pericenter, that can prevent their use for a refined measurement of a relativistic precession.

Therefore, the final error budget of a given relativistic measurement has to account properly for the systematics effects related with both the gravitational and non-gravitational perturbations acting on the orbit of the satellites.

In the following, some of the results that we have recently obtained on the improvements of the models for some of the main NGP acting on the two LAGEOS satellites and on LARES will be introduced.

\subsection{Internal structure and spin dynamics}

In the context of the LARASE activities we reconstructed the dimensions of the two LAGEOS satellites and the materials used to build their internal structure starting from their original drawings ${ }^{55}$, see Figure 2.

The main motivation was to have an independent estimate of their moments of inertia - that were not measured on the flight model of the two satellites - and to have a refined model of the satellites able to provide a reference for the development of a new thermal model in order to account properly of the quite complex perturbation produced by thermal thrust effects. A similar work was performed also for the LARES satellite, see Figure 3. 


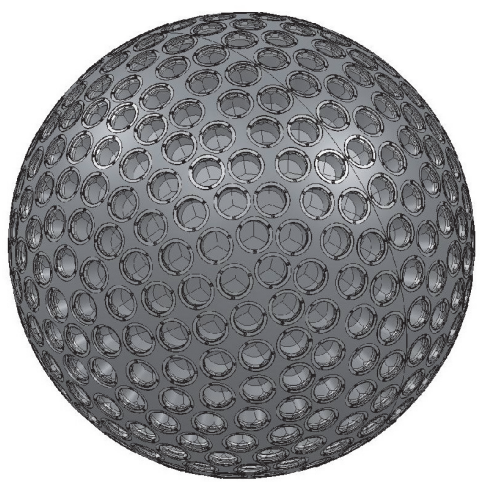

Fig. 2. LARASE model of the LAGEOS II satellite. LAGEOS II is one of the best tracked satellites all over the world by the SLR technique. LAGEOS II is almost twin of the older LAGEOS. The satellites structure is constituted by two hemispheres of aluminum containing 426 cube corner retroreflectors (CCRs), a brass core that contributes to increase the mass of the satellites and a Copper-Beryllium shaft that allows to fasten the different parts of the satellites. The heavy brass core was necessary to guarantee a low area-to-mass ratio for the two satellites, about $6.95 \times 10^{-4}$ $\mathrm{m}^{2} / \mathrm{kg}$, the smallest among the laser-ranged satellites till the recent launch of LARES. LAGEOS II orbit has an inclination of about $53^{\circ}$ over the Earth's equator, a semi-major axis of about $12,163 \mathrm{~km}$ and an eccentricity of about 0.014 . LAGEOS has an orbit inclination of about $110^{\circ}$, a semi-major axis of about $12,270 \mathrm{~km}$ and an eccentricity of about 0.004 . The smaller inclination of LAGEOS II has been chosen to obtain a better visibility from the network of the Earth laser ranging stations.

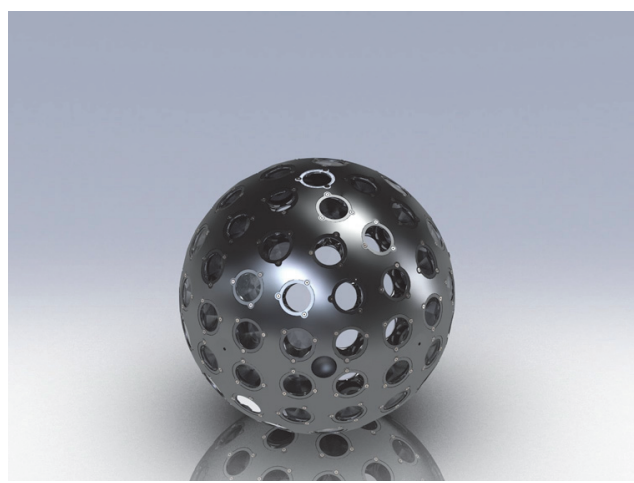

Fig. 3. LARASE rendering of the LARES satellite. LARES is made of a unique piece of tungsten (THA-18N, composition $95 \%$ of $\mathrm{W}$ and $5 \%$ of $\mathrm{Cu}$ and $\mathrm{Ni}$ ) and its surface is covered with $92 \mathrm{CCRs}$ for SLR tracking. The satellite radius is $18.2 \mathrm{~cm}$ and its mass is about $386.8 \mathrm{~kg}$. The CCRs mounting system is quite similar to that of the two LAGEOS. The satellite orbit is almost circular with a semi-major axis of about $7820 \mathrm{~km}$ (corresponding to an orbital period of about $6883 \mathrm{~s}$ ). LARES has been lunched with the qualification flight of the new European launcher VEGA. We refer to ${ }^{9,10}$ for further details.

Moreover, the knowledge of the moments of inertia of a satellite is fundamental in order to model its spin dynamics. Indeed, with regard to the non-gravitational 

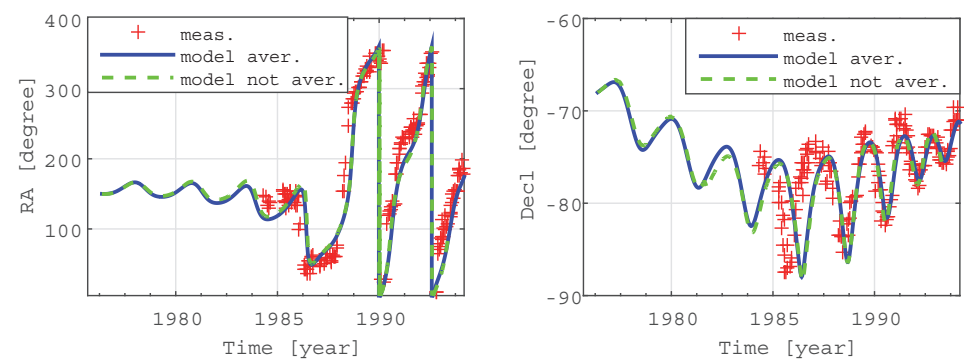

Fig. 4. LAGEOS spin orientation in the J2000 reference frame. Right ascension $\alpha$ (left) and declination $\delta$ (right). The units for the two spherical equatorial coordinates are [deg]. The results for the spin evolution as we obtained from our models - the continuous blue line in the rapidspin case with averaged Eqs., and the green dashed line in the case of the general model - are compared with the available observations in the literature from ${ }^{64}$.

perturbations acting on these satellites, and in the context of the LARASE activities, we have firstly focused our attention on the behaviour of the spin of the considered satellites. Several non-gravitational perturbations depend on the knowledge of the satellite spin vector orientation and rate, among these, the mentioned thermal forces are the most important to consider. Indeed, the rotational dynamics of the two LAGEOS has been deeply investigated in the past by many authors ${ }^{56-62}$.

Beside the knowledge of the moments of inertia of a satellite, which is necessary in order to model the gravitational torque, it is very important to develop a reliable model for the magnetic torque, which also plays a central role. This torque arises because the satellite represents a conductor moving in the Earth's magnetic field. This field induces Foucault currents on the satellite, thence a magnetic moment that, in turn, interacts again with the geomagnetic field and produces the magnetic torque.

In our work we have reviewed deeply the interactions responsible of the spin evolution of the two LAGEOS and we have removed many of the simplifications at the basis of previous models. In particular, we have not simply improved the spin model based on the use of averaged equations for the external torques, starting from the original work by Bertotti and Iess (1991) ${ }^{56}$ and valid in the rapid-spin approximation, but we have been able to develop a completely new model based on the solution of the full set of Euler equations ${ }^{63}$. This work has been also extended to LARES.

In Figures 4 and 5, in the case of the older LAGEOS, the comparison between the evolution of the spin predicted by our models - i.e. the one valid in the rapidspin approximation and the general one valid for any value of the rotational period of the satellite - with the available observations for the the spin of the satellite it is shown. As we can see, the agreements between the two models with the available observations is quite good. 


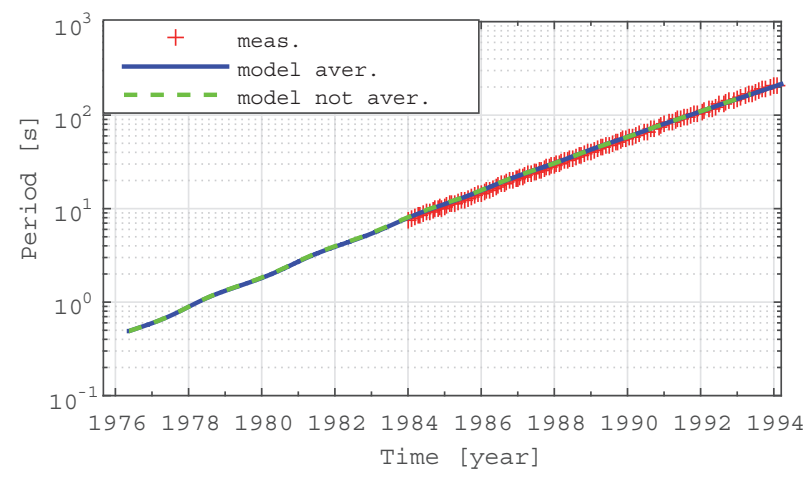

Fig. 5. LAGEOS spin period in the J2000 reference frame. The units for the rotational period $P$ are $[\mathrm{s}]$. The results for the spin evolution as we obtained from our models - the continuous blue line in the rapid-spin case with averaged Eqs., and the green dashed line in the case of the general model - are compared with the available observations in the literature from ${ }^{64}$.

\subsection{Atmospheric neutral drag}

Within the activities of LARASE we reviewed the drag effects on the orbit of the two LAGEOS satellites and we initiated a new study for LARES. Indeed, due to its smaller semi-major with respect to that of the two LAGEOS, the impact of the neutral drag perturbation on the orbit of LARES will be much stronger with respect to its effect on the orbit of the LAGEOS satellites. Among the different activities that we started on this topic, we mainly focused on i) the comparison of the predictions of different atmospheric models at the altitudes of the satellites, ii) the estimate of the perturbing accelerations acting on the satellites and iii) the estimate of the disturbing effects on their orbit. In particular, in our analysis we used a modified version of the SATellite Reentry Analysis Program (SATRAP), a software that is able to load several different models for the Earth's atmosphere together with the appropriate geomagnetic and solar activities indices ${ }^{65,66}$.

Indeed, with SATRAP we have been able to investigate directly the impact of the neutral drag on the satellites orbit using the current best available models for the atmospheres main constituents. In the case of the new LARES satellite, this is also the first step to be performed in order to distinguish the orbital disturbing effects of neutral drag from those of a possible drag due to charged particles and/or from tiny thermal effects. In Figure 6, in the case of the Naval Research Laboratory MSISE-2000 ${ }^{42}$ atmospheric model, we show the comparison for the values of the air density at the heights of the three satellites we are analyzing.

By means of SATRAP we also verified if the decay of the semimajor axis of LARES, that we obtained by a dedicated POD with GEODYN II, was explained in terms of the impact of the neutral drag on the satellite orbit. Indeed, with GEODYN II, we obtained a decay of about $1 \mathrm{~m} / \mathrm{yr}$ for the residuals of the semimajor axis of LARES, that corresponds to a transversal mean acceleration of about $-1.46 \times$ 


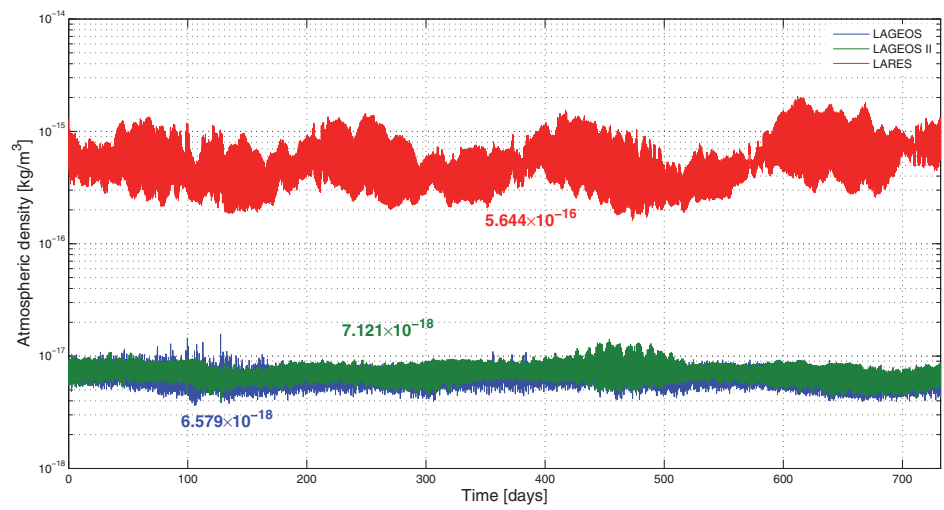

Fig. 6. Variation of the air density $\rho\left[\mathrm{kg} / \mathrm{m}^{3}\right]$ due to the neutral drag at the altitude of the orbits of LAGEOS, LAGEOS II and LARES. The profiles have been obtained from SATRAP using NRLMSISE-2000 to model the behavior of the Earth's atmosphere. The time span covers two years since March 10, 2012. The average densities over the analyzed time span are: $6.6 \cdot 10^{-18}$ $\mathrm{kg} / \mathrm{m}^{3}, 7.1 \cdot 10^{-18} \mathrm{~kg} / \mathrm{m}^{3}$ and $5.6 \cdot 10^{-16} \mathrm{~kg} / \mathrm{m}^{3}$, respectively for LAGEOS, LAGEOS II and LARES.

$10^{-11} \mathrm{~m} / \mathrm{s}^{2}$, see Figure 7 . In the data reduction that we performed, neither the neutral drag nor the thermal effects have been included in the dynamical models of GEODYN II.

With SATRAP the neutral drag perturbation on LARES was computed over the same time span, accounting for the measured decay and considering the real evolution of the solar and geomagnetic activities for several atmospheric models. In particular, assuming as reference for the unmodeled transversal acceleration due to the neutral atmosphere the above value, we obtained that the drag coefficient $C_{D}$ estimated by SATRAP is comparable to the average value estimated by GEODYN II in a least square fit of the tracking data. This means that the current best models developed for the atmosphere behavior are able to account for the observed decay within their errors and range of applicability. In particular, we estimated, using GEODYN II over 7 days arcs, a $C_{D}$ in the range $\approx 3.8-4.0$ - depending on the background model for the neutral atmosphere - in good agreement with the drag coefficients obtained using SATRAP with the same models for the atmosphere of those used in the analyses with GEODYN II.

It is worth of mention that after the estimate of the drag coefficient in order to absorb completely the observed decay, a residual decay was still present, corresponding to an unmodeled (residual) mean transversal acceleration of about $-2 \times 10^{-13}$ $\mathrm{m} / \mathrm{s}^{2}$. Anyway, a further refined analysis is needed in order to confirm, from the observed decay, a possible contribution related with other unmodeled effects, as the thermal ones, which perturb the orbit of the satellite. In this context, it will be necessary to fix the contribution of the drag signature and of the thermal effects in the residuals of the other orbital elements of LARES. 


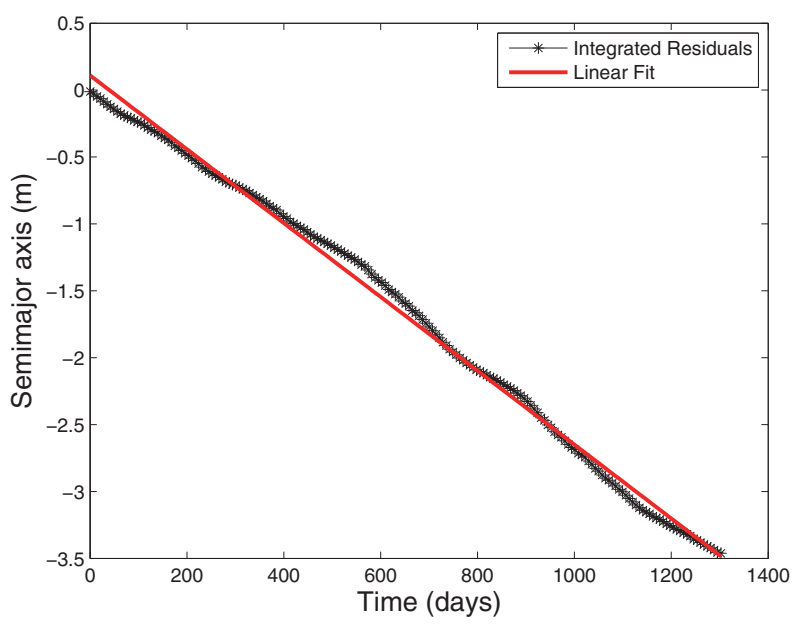

Fig. 7. Decay of LARES semimajor axis residuals [m] over a period of about 3.7-yr since the launch of the satellite. In black are shown the integrated residuals while the continuous red line represents a best linear fit to the data. The measured decay, corresponding to the slope obtained from the linear fit, is $1.008 \mathrm{~m} / \mathrm{yr}$. The data reduction of the satellite orbit was performed with GEODYN II. The neutral drag perturbation was removed from the dynamical model in the case of this particular POD.

\section{Conclusions}

Einstein's GR is today considered as the standard model for the description of the gravitational interaction. However, several theories of physics - not only new gravitational theories, but also those that aim to include GR into the realm of quantum theories - suggest the existence of additional fields in mediating the gravitational interaction to complement the spacetime tensor of GR. Therefore, under the significant theoretical implications that follow from these considerations, new and more refined tests and measurements of gravitation are needed.

The new experiment denominated LARASE (LAser RAnged Satellites Experiment), that we have described in the previous Sections, aims to contribute to these new measurements of relativistic gravity in the WFSM limit of GR. Within LARASE we started an activity to review previous models developed for the two LAGEOS, in particular those related to the non-conservative forces. This activity is also very important in the case of LARES.

We described part of our preliminary results. The study of the internal structure of these satellites and of their spin vector evolution is very important in order to develop new and more reliable models for the thermal effects, especially in the case of the two LAGEOS. The study on the impact of the neutral atmosphere on the orbit of LARES is important in order to fix the possible contribution of smaller effects, as the thermal one and a possible contribution from the drag related with charged particles at the satellite height. 


\section{Acknowledgments}

The authors acknowledge the ILRS for providing high quality laser ranging data of the two LAGEOS satellites and of LARES. This work has been in part supported by the Commissione Scientifica Nazionale II (CSNII) on astroparticle physics experiments of the Istituto Nazionale di Fisica Nucleare (INFN).

\section{References}

1. A. Einstein, Die Grundlage der allgemeinen Relativitätstheorie, Annalen der Physik 354, 769 (1916).

2. C. M. Will, Theory and Experiment in Gravitational Physics (Cambridge University Press, Cambridge, UK, March 1993).

3. A. Einstein, Über das Relativitätsprinzip und die aus demselben gezogenen Folgerungen, Jahrbuch der Radioaktivität und Elektronik 4, 411 (1908).

4. H. M. Schwartz, Einstein's comprehensive 1907 essay on relativity, part III, Am. J. Phys. 45, 899 (October 1977).

5. D. Lucchesi, L. Anselmo, M. Bassan, C. Pardini, R. Peron, G. Pucacco and M. Visco, Testing the gravitational interaction in the field of the Earth via satellite laser ranging and the Laser Ranged Satellites Experiment (LARASE), Class. Quantum Grav. 32, p. 155012 (2015).

6. B. Putney, R. Kolenkiewicz, D. Smith, P. Dunn and M. H. Torrence, Precision orbit determination at the NASA Goddard Space Flight Center, Adv. Space Res. 10, 197 (1990).

7. D. E. Pavlis and et al., GEODYN II Operations Manual. NASA GSFC, (1998).

8. M. R. Pearlman, J. J. Degnan and J. M. Bosworth, The International Laser Ranging Service, Adv. Space Res. 30, 135 (2002).

9. I. Ciufolini, A. Paolozzi, E. C. Pavlis, J. C. Ries, R. Koenig, R. A. Matzner, G. Sindoni and H. Neumayer, Towards a One Percent Measurement of Frame Dragging by Spin with Satellite Laser Ranging to LAGEOS, LAGEOS 2 and LARES and GRACE Gravity Models, Space Sci. Rev. 148, 71 (December 2009).

10. A. Paolozzi and I. Ciufolini, LARES successfully launched in orbit: Satellite and mission description, Acta Astronautica 91, 313 (October 2013).

11. J. Lense and H. Thirring, Phys. Z. 19, p. 156 (1918).

12. B. Mashhoon, F. W. Hehl and D. S. Theiss, On the gravitational effects of rotating masses - The Thirring-Lense Papers, Gen. Rel. Grav. 16, 711 (1984).

13. K. Nordtvedt, Equivalence Principle for Massive Bodies. II. Theory, Phys. Rev. 169, 1017 (1968).

14. C. M. Will, Theoretical Frameworks for Testing Relativistic Gravity. II. Parametrized Post-Newtonian Hydrodynamics, and the Nordtvedt Effect, Astrophys. J. 163, 611 (1971).

15. C. M. Will and J. K. Nordtvedt, Conservation Laws and Preferred Frames in Relativistic Gravity. I. Preferred-Frame Theories and an Extended PPN Formalism, Astrophys. J. 177, 757 (1972).

16. J. K. Nordtvedt and C. M. Will, Conservation Laws and Preferred Frames in Relativistic Gravity. II. Experimental Evidence to Rule Out Preferred-Frame Theories of Gravity, Astrophys. J. 177, 775 (1972). 
17. I. Ciufolini, D. Lucchesi, F. Vespe and A. Mandiello, Measurement of dragging of inertial frames and gravitomagnetic field using laser-ranged satellites, Nuovo Cim. A 109, 575 (1996).

18. I. Ciufolini, F. Chieppa, D. Lucchesi and F. Vespe, Test of Lense - Thirring orbital shift due to spin, Class. Quantum Grav. 14, 2701 (October 1997).

19. I. Ciufolini, D. Lucchesi, F. Vespe and F. Chieppa, Measurement of gravitomagnetism, Europhys. Lett. 39, 359 (1997).

20. I. Ciufolini, E. Pavlis, F. Chieppa, E. Fernandes-Vieira and J. Perez-Mercader, Test of General Relativity and Measurement of the Lense-Thirring Effect with Two Earth Satellites, Science 279, 2100 (1998).

21. I. Ciufolini and E. C. Pavlis, A confirmation of the general relativistic prediction of the Lense-Thirring effect, Nature 431, 958 (2004).

22. D. M. Lucchesi, The Lense-Thirring effect derivation and the LAGEOS satellites orbit analysis with the new gravity field solution from CHAMP, in 35th COSPAR Scientific Assembly, ed. J.-P. Paillé, COSPAR Meeting, Vol. 352004.

23. D. Lucchesi, The LenseThirring effect measurement and LAGEOS satellites orbit analysis with the new gravity field model from the CHAMP mission, Advances in Space Research 39, 324 (2007).

24. D. M. Lucchesi and R. Peron, Accurate Measurement in the Field of the Earth of the General-Relativistic Precession of the LAGEOS II Pericenter and New Constraints on Non-Newtonian Gravity, Phys. Rev. Lett. 105, p. 231103 (December 2010).

25. D. M. Lucchesi and R. Peron, LAGEOS II pericenter general relativistic precession (1993-2005): Error budget and constraints in gravitational physics, Phys. Rev. D 89, p. 082002 (April 2014).

26. C. F. Yoder, J. G. Williams, J. O. Dickey, B. E. Schutz, R. J. Eanes and B. D. Tapley, Secular variation of earth's gravitational harmonic J2 coefficient from Lageos and nontidal acceleration of earth rotation, Nature 303, 757 (June 1983).

27. D. P. Rubincam, Postglacial rebound observed by Lageos and the effective viscosity of the lower mantle, J. Geophys. Res. 89, 1077 (February 1984).

28. S. C. Cohen and D. E. Smith, Lageos scientific results - Introduction, J. Geophys. Res. 90, 9217 (September 1985).

29. D. E. Smith, R. Kolenkiewicz, P. J. Dunn, J. W. Robbins, M. H. Torrence, S. M. Klosko, R. G. Williamson, E. C. Pavlis and N. B. Douglas, Tectonic motion and deformation from satellite laser ranging to Lageos, J. Geophys. Res. 95, 22013 (December 1990).

30. F. G. Lemoine and et al., The Development of the Joint NASA GSFC and the National Imagery and Mapping Agency (NIMA) Geopotential Model EGM96, Technical Paper NASA/TP-1998-206861 (1998).

31. G. Bianco, R. Devoti, M. Fermi, V. Luceri, P. Rutigliano and C. Sciarretta, Estimation of low degree geopotential coefficients using SLR data, Planetary and Space Science 46, 1633 (December 1998).

32. C. M. Cox and B. F. Chao, Detection of a Large-Scale Mass Redistribution in the Terrestrial System Since 1998, Science 297, 831 (August 2002).

33. C. Reigber, R. Schmidt, F. Flechtner, R. König, U. Meyer, K.-H. Neumayer, P. Schwintzer and S. Y. Zhu, An Earth gravity field model complete to degree and order 150 from GRACE: EIGEN-GRACE02S, J. Geodyn. 39, 1 (January 2005). 
34. R. D. Ray, A Global Ocean Tide Model From TOPEX/POSEIDON Altimetry: GOT99.2, Technical Paper NASA/TM-1999-209478, Goddard Space Flight Center, Greenbelt, Maryland (1999).

35. G. Petit and B. Luzum, IERS Conventions (2010), IERS Technical Note 36, IERS (Frankfurt am Main: Verlag des Bundesamts für Kartographie und Geodäsie, 2010).

36. E. M. Standish, X. X. Newhall, J. G. Williams and W. M. Folkner, JPL Planetary and Lunar Ephemerides, DE403/LE403, Tech. Rep. JPL IOM 314.10-127 (1995).

37. C. Huang, J. C. Ries, B. D. Tapley and M. M. Watkins, Relativistic effects for nearearth satellite orbit determination, Celest. Mech. Dyn. Astron. 48, 167 (1990).

38. D. P. Rubincam, P. Knocke, V. R. Taylor and S. Blackwell, Earth anisotropic reflection and the orbit of LAGEOS, J. Geophys. Res. 92, 11662 (October 1987).

39. D. P. Rubincam, LAGEOS orbit decay due to infrared radiation from earth, J .Geophys. Res. 92, 1287 (February 1987).

40. D. P. Rubincam, Yarkovsky thermal drag on LAGEOS, J. Geophys. Res. 93, 13805 (November 1988).

41. D. P. Rubincam, Drag on the Lageos satellite, J. Geophys. Res. 95, 4881 (April 1990).

42. J. M. Picone, A. E. Hedin, D. P. Drob and A. C. Aikin, NRLMSISE-00 empirical model of the atmosphere: Statistical comparisons and scientific issues, J. Geophys. Res. 107, p. 1468 (December 2002).

43. Z. Altamimi, X. Collilieux and L. Métivier, ITRF2008: an improved solution of the international terrestrial reference frame, J. Geod. 85, 457 (August 2011).

44. International Earth Rotation Service, EOP Combined Series EOP C04, tech. rep., IERS.

45. D. M. Lucchesi and G. Balmino, The LAGEOS satellites orbital residuals determination and the Lense Thirring effect measurement, Plan. Space Sci. 54, 581 (2006).

46. G. Afonso, F. Barlier, F. Mignard, M. Carpino and P. Farinella, Orbital effects of LAGEOS seasons and eclipses, Ann. Geophysicae 7, 501 (October 1989).

47. P. Farinella, A. M. Nobili, F. Barlier and F. Mignard, Effects of thermal thrust on the node and inclination of LAGEOS, Astron. Astrophys. 234, 546 (August 1990).

48. R. Scharroo, K. F. Wakker, B. A. C. Ambrosius and R. Noomen, On the along-track acceleration of the Lageos satellite, J. Geophys. Res. 96, 729 (January 1991).

49. V. J. Slabinski, A Numerical Solution for Lageos Thermal Thrust: The Rapid-Spin Case, Celest. Mech. Dyn. Astron. 66, 131 (June 1996).

50. P. Farinella and D. Vokrouhlický, Thermal force effects on slowly rotating, spherical artificial satellites-I. Solar heating, Plan. Space Sci. 44, 1551 (December 1996).

51. D. P. Rubincam, D. G. Currie and J. W. Robbins, LAGEOS I once-per-revolution force due to solar heating, J. Geophys. Res. 102, 585 (January 1997).

52. G. Métris, D. Vokrouhlický, J. C. Ries and R. J. Eanes, Nongravitational effects and the LAGEOS eccentricity excitations, J. Geophys. Res. 102, 2711 (February 1997).

53. G. Métris, D. Vokrouhlický, J. C. Ries and R. J. Eanes, LAGEOS Spin Axis and Non-gravitational Excitations of its Orbit, Adv. Space Res. 23, 721 (1999).

54. D. M. Lucchesi, Reassessment of the error modelling of non-gravitational perturbations on LAGEOS II and their impact in the Lense-Thirring derivation-Part II, Plan. Space Sci. 50, 1067 (August 2002).

55. M. Visco and D. Lucchesi, Review and critical analysis of mass and moments of inertia of the LAGEOS and LAGEOS II satellites for the LARASE program, Submitted to Advances Space Res. (2016). 
56. B. Bertotti and L. Iess, The rotation of Lageos, J. Geophys. Res. 96, 2431 (February 1991).

57. S. Habib, D. E. Holz, A. Kheyfets, R. A. Matzner, W. A. Miller and B. W. Tolman, Spin dynamics of the LAGEOS satellite in support of a measurement of the Earth's gravitomagnetism, Phys. Rev. D 50, 6068 (November 1994).

58. P. Farinella, D. Vokrouhlicky and F. Barlier, The rotation of LAGEOS and its longterm semimajor axis decay: A self-consistent solution, J. Geophys. Res. 101, 17861 (August 1996).

59. D. Vokrouhlický, Non-gravitational effects and LAGEOS' rotation, Geophys. Res. Lett. 23, 3079 (1996).

60. S. E. Williams, The Lageos Satellite: A Comprehensive Spin Model and Analysis, PhD thesis, NCSU PhD Dissertation, pp. i-xii, 1-252, 2002 December 2002.

61. J. I. Andrés, R. Noomen, G. Bianco, D. G. Currie and T. Otsubo, Spin axis behavior of the LAGEOS satellites, J. Geophys. Res. 109, p. 6403 (June 2004).

62. J. I. Andrés de la Fuente, Enhanced Modelling of LAGEOS Non-Gravitational Perturbations, PhD thesis, Delft University Press, (Sieca Repro, Turbineweg 20, 2627 BP Delft, The Netherlands, 2007).

63. M. Visco and D. Lucchesi, SPIN, TBS (2016).

64. D. Kucharski, H.-C. Lim, G. Kirchner and J.-Y. Hwang, Spin parameters of LAGEOS1 and LAGEOS-2 spectrally determined from Satellite Laser Ranging data, Adv.Space Res. 52, 1332 (October 2013).

65. C. Pardini and L. Anselmo, SATRAP: Satellite reentry analysis program, Internal Report C94-17, CNUCE Institute, Consiglio Nazionale delle Ricerche (CNR) (Pisa, Italy, 1994).

66. C. Pardini, K. Moe and L. Anselmo, Thermospheric density model biases at the 23rd sunspot maximum, Plan. Space Sci. 67, 130 (July 2012). 